\title{
Resonantly-enhanced axion-photon regeneration
}

\author{
Guido Mueller, ${ }^{*}$ Pierre Sikivie, ${ }^{*}$ David B. Tanner, ${ }^{*}$ and Karl van Bibber ${ }^{\dagger}$ \\ ${ }^{*}$ Department of Physics, University of Florida,Gainesville, FL 32611, USA \\ ${ }^{\dagger}$ Naval Postgraduate School, Monterey, CA 93943, USA
}

\begin{abstract}
.
A resonantly-enhanced photon-regeneration experiment to search for the axion or axion-like particles is discussed. Photons enter a strong magnetic field and some are converted to axions; the axions can pass through an opaque wall and some may convert back to photons in a second high-field region. The photon regeneration is enhanced by employing matched Fabry-Perot optical cavities, with one cavity within the axion generation magnet and the second within the photon regeneration magnet. The optics for this experiment are discussed, with emphasis on the alignment of the two cavities.
\end{abstract}

Keywords: axion, photon regeneration, shining light through walls

PACS: $12.38 . \mathrm{Qk}, 14.80 . \mathrm{Mz}, 29.90 .+\mathrm{r}$

The axion remains the most attractive solution to the strong-CP problem and is also one of two leading dark-matter candidates.[1,2] Present constraints restrict the axion mass $m c^{2}$ to $1 \mu \mathrm{eV}-15 \mathrm{meV}$, making it very weakly interacting. Nevertheless, there is considerable ongoing experimental effort to search for axions. All rely on the coupling of axions to two photons; moreover, all are based on the Primakoff effect where one of the photons is virtual. An axion can convert into a single real photon of the same energy (or vice versa) in the presence of a strong magnetic field.[3]

The simplest purely laboratory experiment to look for axions is photon regeneration.[4-11] The sensitivity of the experiment, described below, is poor in its basic form, improved only by increasing the magnetic field strength or the length of the interaction regions. However, very large gains may be realized by introducing matched optical resonators in both the axion production and the photon regeneration regions.[12-14] This paper describes some practical details of such an experiment, focusing on the scheme for aligning and matching the two cavities. For background, including a discussion of the physics of the conversion process, the resonant gain, discussion of scheme for locking two matched high-finesse optical resonators, the signal detection method, and the ultimate noise limits, we refer readers to our earlier paper.[14] We will consider two searches: Phase I uses 2.5 meter cavities and $0.6 \mathrm{~T}$ permanent magnets while Phase II, a much more demanding search, uses 44-m long cavities and 5 T superconducting magnets. Phase I would improve on present limits by a factor of $8-10$ and Phase II by a factor of more than 10,000 . We note also that both experiments, although challenging, are feasible using technologies developed for laser interferometer gravitational-wave detectors.[15, 16]

Figure 1a shows the photon regeneration experiment as usually conceived. The number of regenerated photons is $N_{S}=P_{\gamma} P_{a} N_{\text {in }}$ where $N_{\text {in }}$ is the number of photons in the initial laser beam, $P_{\gamma}$ is the photon-to-axion conversion probability in the magnet on the 


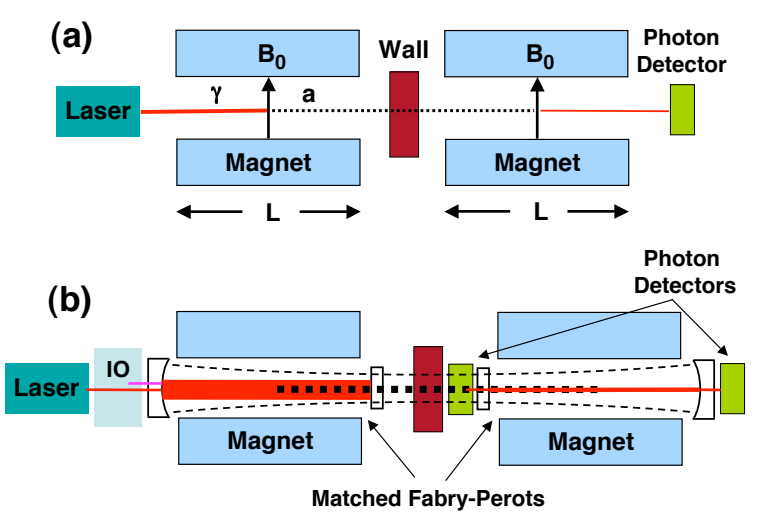

FIGURE 1. (a) Simple photon regeneration. (b) Resonant photon regeneration, employing matched Fabry-Perot cavities. The envelope schematically shown by the thin dashed lines indicates the important condition that the axion wave, and thus the mode, in the photon regeneration cavity must follow that of the hypothetically unimpeded photon wave from the mode in the axion generation magnet.

LHS and $P_{a}$ is the axion-to-photon conversion probability in the magnet on the RHS.

Figure $1 \mathrm{~b}$ shows two improvements[12-14] for the experiment. The first is to build up the electric field on the left hand side of the experiment using a Fabry-Perot cavity, the axion generation cavity. Axions created in the generation cavity propagate through the "wall" and reconvert into photons in the regeneration region. The second improvement is to install also a second Fabry-Perot cavity, the photon regeneration cavity, on the other side of the experiment, making a symmetric arrangement. The final signal depends on the laser power build-up in the axion generation cavity and the signal build-up in the photon regeneration cavity. As shown in reference[14] the final signal depends on the finesse $\mathscr{F}_{\gamma}$ and $\mathscr{F}_{a}$ of the generation and regeneration cavities, respectively.

$$
N_{S}=\eta^{2} \frac{\mathscr{F}_{\gamma}}{\pi} \frac{\mathscr{F}_{a}}{\pi} P_{\gamma} P_{a} N_{\text {in }}
$$

where $\eta$ is the spatial overlap integral between the axion mode and the electric field mode, i.e., the efficiency of coupling of the two cavities.. Resonant regeneration gives an enhancement factor of $\sim(\mathscr{F} / \pi)^{2}$ over simple photon regeneration. This factor may feasibly be $10^{10}$, corresponding to an improvement in sensitivity to the coupling of axions to two photons, $g_{a \gamma \gamma}$, of $\sim 300$.

The resonantly-enhanced photon regeneration experiment, involving the design and active locking of high-finesse Fabry-Perot resonators and the heterodyne detection of weak signals at the shot-noise limit, is well supported by the laser and optics technology developed for LIGO, as discussed in Ref. [14].

The experiment requires that the optical cavities support mirror-image fundamental spatial modes and have a common waist location; consequently the outer mirrors are curved and the inner ones are essentially flat. In addition, losses due to aperture effects should be kept very low, requiring not too big a divergence of the light away from the waist. These considerations, together with the dimensions of the available magnets, drive 
the design, leading to $g$-parameters[17] for the optical cavities such that $g=g_{1} g_{2}=0.6$, where $g_{i}=1-L_{c a v} / R_{i}$, with $L_{c a v}$ the cavity length and $R_{i}$ the radii of curvature of the mirrors. Absorption values at $1064 \mathrm{~nm}$ of less than $1 \mathrm{ppm} / \mathrm{bounce}$ are available from a number of vendors,[18] allowing a cavity finesse of well above the anticipated value of 300,000 during the Phase II experiments. However, the development of a robust length/frequency and alignment sensing and control scheme will greatly be accelerated if we start Phase I experiments with a modest transmissivity of $T=1000 \mathrm{ppm}$ (finesse of $\approx 6000$ ), and later replace the mirrors to increase the finesse.

The efficiency of photon regeneration will depend on alignment mismatches between the cavities. Losses caused by a lateral shift $\delta x$ scale with the beam size $w$; those caused by an angular shift $\delta \alpha$ scale with the divergence angle $\Theta$, making the overall efficiency

$$
\eta \approx 1-\frac{1}{2} \frac{\delta x^{2}}{w^{2}}-\frac{1}{2} \frac{\delta \alpha^{2}}{\Theta^{2}} \quad \text { with }: \quad \Theta=\frac{\lambda}{\pi w}
$$

Requiring an efficiency $\eta>0.95$, we find for Phase I:

$$
\delta x<\sqrt{0.05} w_{1} \approx 0.2 \mathrm{~mm} \quad \delta \alpha<\sqrt{0.05} \Theta \approx 50 \mu \mathrm{rad}
$$

For the Phase II TeV 6+6 configuration the requirements are:

$$
\delta x<1 \mathrm{~mm} \quad \delta \alpha<10 \mu \mathrm{rad}
$$

One of the main challenges of the experiment is to align correctly the two cavities and to maintain the alignment throughout the experiment. Recall that it is the axion field which couples the two cavities and that axions, in contrast to light beams, do not experience any refraction in matter. In addition, we have to avoid any leakage of photons from the axion generation cavity into the photon regeneration cavity. Both conditions limit the number of possibilities for alignment sensing and control. Developing and testing the length and alignment sensing and control scheme is the reason why we believe that Phase I is necessary.

Figure 2 shows the proposed experimental setup. This apparatus is designed to meet the requirement that the axion generation and photon regeneration cavities are both on resonance and are aligned with the axion generating laser field. In the following paragraphs we discuss the initial alignment of the experiment.

In contrast to all other degrees of freedom, the distance between and the alignment of FM1 and FM2 will not be actively controlled but will depend on the initial alignment and passive stability of the central bench. Therefore, the assembly of the central bench is one of the most critical steps. The goal is to ensure that the surfaces of the two flat mirrors FM1 and FM2 which face the curved mirrors CM1 and CM2 are parallel to better than $10 \mu \mathrm{rad}$ and that their separation does not change by more than a fraction of the laser wavelength during the entire course of the experiment.

The central plate will be made from a ultra-low coefficient of thermal expansion material such as Zerodur or ULE.[19] The mirrors themselves will be aligned and optically contacted to the central plate. The alignment procedure uses two identical curved mirrors with a transmissivity of $T \approx 1 \%$. These will be placed about $2 \mathrm{~m}$ apart from each other on an optical table in a clean room. (See Fig. 3). These mirrors form a 


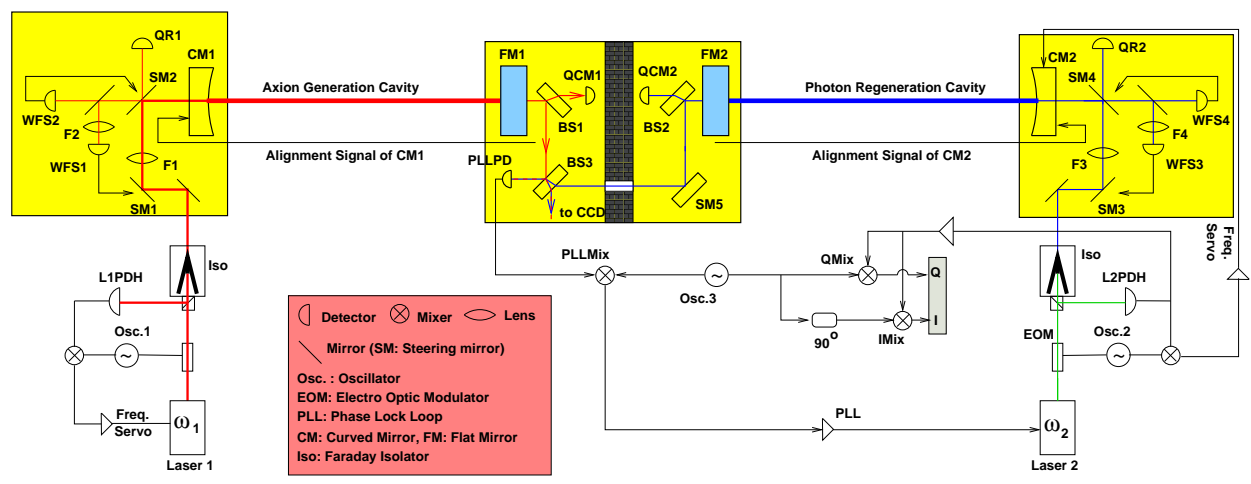

FIGURE 2. Experimental Layout: The left cavity formed between CM1 and FM1 produces axions from the electric field of Laser 1 . The right cavity is used to regenerate the electric field from the axions which enter it. The layout shows the detectors and sensors needed for length, frequency, and alignment control.

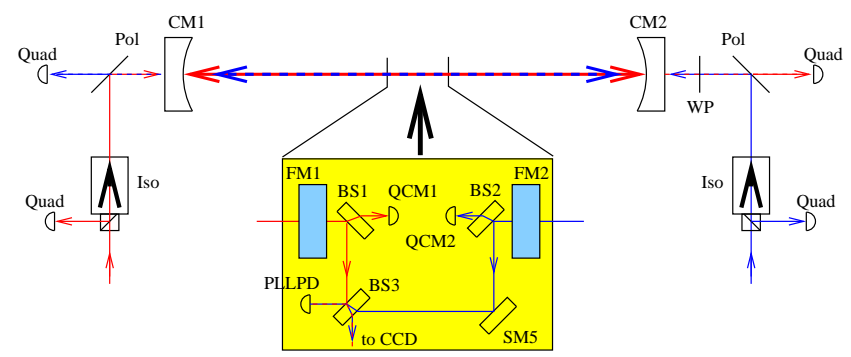

FIGURE 3. The alignment procedure for the central plate uses an optical cavity formed between two curved mirrors CM1 and CM2 as a reference. This cavity will be interrogated by two laser beams (red and blue) injected from opposite sites into the cavity. FM1/2: Flat mirrors, QCM1/2: Position sensitive Quadrant detectors.

low finesse optical cavity and define the optical axis during the alignment procedure. One laser field (red) will be injected from the left into the cavity. The positions of the reflected field and the transmitted field will be monitored with position-sensitive detectors (Quads, or quadrant photodiodes), which also measure the total incident power. The half-wave plate (WP) turns the s-polarized light into p-polarized light and allows transmission through the polarizer to the Quad behind CM2. The frequency of the laser will be scanned and the alignment and mode matching will be optimized by maximizing the transmission of the fundamental spatial mode and minimizing all other modes. A second laser (blue) will also be aligned and mode matched into the cavity and its reflected and transmitted beam position will be monitored with two additional Quads.

Two beam splitters (BS1, BS2) and two additional Quads (QCM1, QCM2) will be installed on the central board and then the board will be placed between the two cavities. Note that the refraction inside the beam splitter separates the optical axis from the propagation axis of axions. The Quad signals will be used to verify that we can reproducibly move the central board in and out of its position. 
Once repeatability has been verified, the position of FM1 will be adjusted until the cavity formed with CM1 is well aligned to the red laser beam. This process will then be repeated for FM2 with respect to CM2 and the blue laser. After removal and replacement, to ensure that the alignment of the curved mirrors and of the two laser beams is still good, FM1 and FM2 are optically contacted to the bench in their proper alignment. Note that similar alignment procedures have been developed for the optical bench of the LISA Pathfinder mission.[20] Then the central board will be moved back into place and the waveplate (WP) will be removed. We will align and optically contact the steering mirror SM5 and beam splitter BS3 to generate a beat signal between the blue and the red laser on the single element detector PLLPD. The second output of the BS3 will later be used to monitor the beam profiles on a CCD camera. After this, the surfaces of the two flat mirrors will be parallel to each other. Consequently, the optical axis of the two cavity eigenmodes will later be parallel to each other.

We will then assemble the two breadboards holding the curved mirrors CM1 and 2, SM1-4, F1 and F3. The transmission of the curved mirrors will initially be around 1000 ppm. CM1 and CM2 will be mounted or suspended in a way that their longitudinal position and their angular orientation can be adjusted as part of a feedback loop. Similarly, the alignment of the steering mirrors SM1 to 4 can be actively controlled. These breadboards will then be placed inside the vacuum tanks at the end of each magnet. All other optical components, detectors, the lasers, modulators, and isolators will be mounted in standard mirror or lens mounts on these optical tables without any active actuators as shown in Figure 2.

The initial alignment of the actual experiment is similar to the alignment procedure used to assemble the central bench. First we will remove the central bench and the beam block to form a cavity between CM1 and CM2. Laser 1 will then be aligned to pass through the modulator and isolator before it is injected into this cavity. Similarly, Laser 2 passes through its modulator and isolator and is injected into the cavity from the other side, Once the laser beams are aligned to the cavity formed between CM1 and CM3, the alignment of CM1 and CM2 will then be stabilized to additional optical lever signals (not shown) which are unaffected by the resonant build-up inside the cavity. A small amount of light from laser 1 will leak through SM2; some from laser 2 will leak through SM4. These fields will be detected by the quad detectors QR1 and QR2, allowing us to monitor the position of the input beam. The angular control of the cavity reflected light will be accomplished with wavefront sensors (WFS1,2). Each WFS consists of a quadrant photodetector, with four quarter-circle elements, one in each Cartesian quadrant. The differences in the photocurrent between the elements of the WFS will be used to control the alignment of the laser beam while the cavities are non-resonant. Then we move the central bench back into place and align it using mechanical feedthroughs until both cavities are aligned with respect to the laser beams. Then, once again, we remove the central bench to verify that CM1, CM2, and the lasers are still aligned. This process will be iterated until we are certain that the central bench is well aligned with the curved mirrors and the laser fields.

The lengths of each cavity and the alignment of the end curved mirrors will be controlled using the Pound-Drever-Hall technique.[21] The control hierarchy is that the wavelength of Laser 1 is locked to the length of the axion generation cavity, Laser 2 is offset locked to Laser 1 by a multiple of the cavity free spectral range, and finally the 
length of the photon regeneration cavity is locked to the wavelength of Laser 2.

One of the differences between the experimental layout proposed here and the design described in Ref. [14] is that the lasers enter both cavities through the curved mirrors at the far ends and not through the flat mirrors in the center. This allows us to remove the central bench to verify the overall alignment of the two cavities. A second advantage is that the final detector L2PDH is now far away from Laser 1, reducing significantly the chances of contamination of the signal with stray light. The only optical connection is through the cavities while all other beam paths can easily be blocked. A third advantage is flexibility: the finesse of both cavities depends mainly on the transmissivity of the input or curved mirror. The design described above allows us to change these mirrors without affecting the critical alignment of the two central mirrors.

This work was supported by the U.S. Department of Energy under contracts DEFG02-97ER41029, and DE-AC52-07NA27344.

\section{REFERENCES}

1. M.S. Turner, Phys. Rep. 197, 67 (1990); G.G. Raffelt, Phys. Rep. 198, 1 (1990).

2. Jihn E. Kim and Gianpaolo Carosi, Rev. Mod. Phys. 82, 557 (2010).

3. P. Sikivie, Phys. Rev. Lett. 51, 1415 (1983).

4. K. van Bibber, N.R. Dagdeviren, S.E. Koonin, A.K. Kerman and H.N. Nelson, Phys. Rev. Lett. 59, 759 (1987).

5. G. Ruoso, R. Cameron, G. Cantatore, A.C. Melissinos, Y. Semertzidis, H.J. Halama, D.M. Lazarus, A.G. Prodell, F. Nezrick, C. Rizzo and E. Zavattini, Z. Phys. C 56, 505 (1992).

6. R. Cameron, G. Cantatore, A.C. Melissinos, G. Ruoso, Y. Semertzidis, H.J. Halama, D.M. Lazarus, A.G. Prodell, F. Nezrick, C. Rizzo, and E. Zavattini, Phys. Rev. D 47, 3707 (1993).

7. C. Robilliard, R. Battesti, M. Fouché, J. Mauchain, A.-M. Sautivet, F. Amiranoff, and C. Rizzo, Phys. Rev. Lett. 99190403 (2007).

8. A.S. Chou, W. Wester, A. Baumbaugh, H.R. Gustafson, Y. Irizarry-Valle, P.O. Mazur, J.H. Steffen, R. Tomlin, X. Yang, and J. Yoo, Phys. Rev. Lett. 100080402 (2008).

9. A. Afanasev, O.K. Baker, K.B. Beard, G. Biallas, J. Boyce, M. Minarni, R. Ramdon, M. Shinn, and P. Slocum, Phys. Rev. Lett. 101120401 (2008)

10. P. Pugnat, L. Duvillaret, R. Jost, G. Vitrant, D. Romanini, A. Siemko, R. Ballou, B. Barbara, M. Finger, M. Finger, J. Hošek, M. Král, K.A. Meissner, M. Šulc, and J. Zicha, Phys. Rev. D 78, 092003 (2008)

11. K. Ehret, M. Frede, S. Ghazaryan, M. Hildebrandt, E,A. Knabbe, D. Kracht, A. Lindner, J. List, T. Meier, N. Meyer, D. Notz, J. Redondo, A. Ringwald, G. Wiedemann, and B. Willke, Nucl. Instrum. Meth. A612, 83 (2009); Physics Letters B 689, 149 (2010).

12. F. Hoogeveen and T. Ziegenhagen Nucl. Phys. B 358, 3 (1991).

13. P. Sikivie, D.B. Tanner, and Karl van Bibber, Phys. Rev. Lett. 98, 172002 (2007).

14. G. Mueller, P. Sikivie, D.B. Tanner and K. van Bibber, Phys. Rev. D 80, 072004 (2009).

15. B. Abbott et al. (LIGO Scientific Collaboration), Nucl. Instrum. Methods A 517, 154-179 (2004); Rep. Prog. Phys. 72, 076901/1-25 (2009).

16. R.J. Cruz, J.I. Thorpe, A. Preston, R. Delgadillo, M. Hartman, S. Mitryk, A. Worley, G. Boothe, S. R. Guntaka, S. Klimenko, D.B. Tanner and G. Mueller, Class. Quant. Grav. 23, S751-S760 (2006).

17. A.E. Siegman, Lasers (University Science Books, Sausalito, 1984).

18. For example, see http://lma.in2p3.fr/Activites/loss.htm

19. Zerodur is a glass-ceramic made by Schott; ULE is a glass-ceramic made by Corning.

20. C.J. Killow, J. Bogenstahl, F. Guzmán Cervantes, M. Perreur-Lloyd, D.I. Robertson, F. Steier, and H. Ward, AIP Conf. Proc. 873, 297 (2006).

21. R.W.P. Drever, J.L. Hall, F.V. Kowalski, J. Hough, G.M. Ford, A.J. Munley, and H. Ward, Appl. Phys. B 31, 97 (1983); Eric D. Black, Am. J. Phys. 69, 79 (2001). 